\title{
REGION
}

The Journal of ERSA

Powered by WU

Volume 1, Number 1, 2014, R1

journal homepage: region.ersa.org

\section{Spatial data, analysis, and regression - a mini course}

\author{
Daniel Arribas-Bel ${ }^{1}$ \\ ${ }^{1}$ School of Geography, Earth and Environmental Sciences, University of Birmingham, Birmingham, \\ United Kingdom (email: d.arribas-bel@bham.ac.uk)
}

Received: 20 October 2014/Accepted: 21 October 2014

\begin{abstract}
This resource contains the materials and structure suggested to run a mini course of approximately 14 hours on spatial data, analysis and regression. The course is structured along four lectures and four labs that require the use of computers.
\end{abstract}

Key words: spatial analysis, course, open-source

\section{Description of the Resource}

Lectures present an introductory overview of why it is important to explicitly consider space in quantitative analysis. The first session covers different types of spatial data and motivates spatial analysis, introducing the concept of spatial dependence and stressing its differences with spatial heterogeneity. The next session introduces spatial weights, the spatial lag operator and provides an overview of the most basic tools of exploratory spatial data analysis (ESDA); the third and fourth lectures delve into spatial regression. After some statements motivating the topic, time is spent on model specification, diagnostics and estimation, and concludes with an overview of software implementations of spatial econometric techniques.

Computer labs provide practical lessons that solidify the concepts explained in the lectures and allow the student to learn some of the main tools available to carry out spatial analysis. The first session uses QGIS to open, manipulate and transform spatial data. The second lab uses GeoDa as an interactive tool to explore data and perform the main ESDA techniques. The third lab covers the specification and estimation of spatial econometric models using GeoDaSpace, while the fourth replicates its results using the open-source Python library PySAL.

As a whole, this resource is intended for both instructors and students. The latter can follow the structure of the sessions, get a sense of the main topics through the slides provided and continue with the suggested readings. The former can use it as an initial set of material and adapt it to their own teaching practices, extending areas considered more relevant, or skipping parts deemed unnecessary for their own needs. To that end, the course is released as an open-source software project and licensed using CreativeCommons, which allows reuse, remix and redistribution.

\section{Resource links}

- Website: http://darribas.org/sdar_mini

- Materials: https://github.com/darribas/sdar_mini/releases/tag/v1.0 